HADRODUCTION OF HEAVY FLAVORS

I . D. Leedom

Apri1, 1986 


\title{
Hadroduction of Heavy Flavors
}

\author{
Ian D. Leedom
}

Fermilab

The subject of this review, as one can gather from the title, is the current state of knowledge (or ignorance) of heavy quark production, particularly charm, by hadron beams. The only report of $B$ mesons in a hadron experiment is that of the WA75 collaboration. (1) We shall come back to that experiment because of its interesting implications for $B$ lifetimes, but the rest of the paper will deal exclusively with charm production.

Charm hadroproduction, I ike all Gaul, can be divided into three parts: total cross section, $p_{T}$ and $x_{F}$ dependence. The only other question that arises is the species which have been observed. Of the mesons, the charged and neutral $D$ and $D^{*}$ have certainly been seen. $(2,3,4)$ The $F$ has probably been found ${ }^{(5)}$ but the evidence is sketchy and somewhat contradictory. The $\Lambda_{c}$ and the $A^{+}$baryons appear secure, $(6,7,8)$ while the $T^{0}$ is on much shakier ground. (9)

\section{Cross Sections}


Quoted charm cross sections are large, but there exist great uncertainties as to how large. The reason for this is due primarily to: uncertainties in nuclear cross sections and the extrapolation to hydrogen; large and difficult acceptance and efficiency calulations; and poorly known exclusive branching ratios. As $D$ production is the best known, we begin with it. The published values are shown in Table 1 .

Most hadron-nucleon charm cross sections have been derived from results on heavy nuclei. Such cross sections are normally calculated by assuming that

$$
\sigma_{A}=A^{\alpha} \sigma_{p}
$$

with $\alpha$ a constant. If heavy quarks are formed in hard scattering processes, then one expects that the exponent should be equal to unity. This would be in agreement with $\mathrm{J} / \Psi$ production. The E613 collaboration has measured prompt $\nu$ fluxes, which presumably come from charm decays, on three different target materials: beryllium, copper and tungsten. (10) The best fit gives $\alpha=0.75$. The inferred cross section of $28.6 \mu \mathrm{b}$./nucleon is twice as large as that found by the NA27 collaboration using the hydrogen bubble chamber LEBC. (11) In fact the beam dump values shown in Table 1 have been calculated using $\alpha=1$ so that they agree with the LEBC data. The smaller value of $\alpha$ found by E613 
would give a consequent increase in these cross sections by factors of $1.7,2.7$ and 3.7 for beryllium, iron and tungsten respectively.

The question of the relation of $p-p$ to $p$-nucleus cross sections was investigated by Barton et al. (12) for the production of strange and non-strange species. Figure 1 shows their findings for $\alpha$ as a function of $x_{F}$. They showed that cross sections on nuclei extrapolate to larger proton cross sections than are measured in hydrogen and that $\alpha$ is a function of $x$ independent of beam or product type. A recent CERN experiment, ${ }^{(13)}$ however, seems to indicate that a may depend on the produced species. What is certain is that the simple assumption embodied in Eq. 1 is naive and that charm production on nuclei is a much more complicated process than was anticipated. One is led to conclude that the only reliable cross sections are those found using proton targets.

This only leaves the ISR and LEBC results. The latter are small statistics experiments (NA16 and NA27), but with very clean, reliable data samples and cross sections which are inferred from topological rather than exclusive decay channels. The ISR experiments typically have correction factors $210^{6}$ and depend critically on exclusive branching ratios into the observed final states. These cross sections have been calculated using values of the $D$ branching ratios 
which have changed by factors of 2-4, depending on the mode, over the past 8 years. (14) The ISR results cannot, therefore, be considered to give reliable cross sections. The only known cross sections for D production are the LEBC measurements for pions at $\sqrt{s}=26 \mathrm{GeV}$ and protons at $\sqrt{s}=27$ GeV.

The ACCMOR collaboration (NA11) has published evidence for F production in $\pi^{-} \mathrm{Be}$ reactions. (5) The signal sought is the $\phi \pi$ decay mode as is seen in $e^{+} e^{-}$reactions. Data from both $\phi$ and electron triggers were used. F and Cabbibo suppressed D decays are seen at approximately the same rate. The data reduction takes $5 \times 10^{6}$ triggers to yield 14 events in the final sample of which 5 are $F$ candidates, 3 of these being ambiguous with a $\Lambda_{c}$ interpretation. The calculated lifetime is $\tau_{F}=3.2_{-1.2}^{+3.0} \times 10^{-13}$. The Fermilab experiment E623 also looked for $F$ production in the $\phi \pi$ final state. (15) While the decay of the charged D is easily seen (Fig. 2), there is no enhancement in the $F$ region. Additionally, NA27 has set a limit on $F$ production in $\pi p$ interactions by searching for decays in which the outgoing $K$ is of the same sign as the decaying particle. This would correspond to either Cabbibo suppressed D or allowed F decay. No signal is seen at the level of $750 \mathrm{nb}$. (16) It should be noted that this limit assumes an F lifetime greater than $2 \times 10^{-13}$ s. A shorter $F$ I ifetime would imply a less restrictive limit. More information is needed before the $F$ can claim to have been seen in hadronic reactions. 
Unlike the $F$, there is no doubt that the $\Lambda_{c}$ is produced hadronically. The first reports came from the $\operatorname{ISR}^{(6,7)}$ and it has since been seen both in SPS experiments (e.g. NA27) with $\pi$ and proton beams and in a neutron on Carbon experiment at $\sqrt{s}$ of $9 \mathrm{GeV}$ (17) The ISR cross sections for $\Lambda_{c}$ production suffer from extreme model dependence due to the restrictive triggers used and I imited apparatus acceptance. All of the experiments are plagued with very poorly known branching ratios. In most cases, the exclusive decay modes are known to no better than $50 \%$. Therefore it is best to say that the $\Lambda_{c}$ cross section is unknown at any energy.

The $\mathrm{A}^{+}$baryon (quark content:csu) has been reported by the WA42 collaboration. $(8,9)$ The experment used a $135 \mathrm{GeV} \Sigma^{-}$ beam on a Be target, but because the spectrometer is only sensitive to $x_{F}>0.6$, the calculated cross section is highly dependent on the apparatus acceptance to the $\Lambda K^{-} \pi^{+} \pi^{+}$final state. The same group also claims evidence for the $T^{0}$ (quark content:css) but with much lower significance. Both observations require confirmation.

To summarize our knowledge of charm cross sections.

$$
\begin{array}{ll}
* \sigma_{p p}(D \bar{D})=11 \cdot 2 \pm 2.3 \mu \mathrm{b} . & x_{F}>0 \sqrt{s}=27 \mathrm{GeV} \\
* \sigma_{\pi p}(D \bar{D})=7.9 \pm 1 \cdot 3 \mu \mathrm{b} . & x_{F}>0 \sqrt{s}=26 \mathrm{GeV}
\end{array}
$$


$* \sigma_{\pi p}(F) \leq(750 \pm 250) \mathrm{nb} . \quad x_{F}>0 \quad \tau_{F}>2.0 \times 10^{-13} \mathrm{~s}$.

The unfortunate side of the coin is what we do not know.

- any of the above cross section at other energies.

- the $\Lambda_{c}$ cross section at any energy at all.

- how to convert $p$-nucleus to $p$-nucleon cross sections.

The last point is one of the most telling. Until we can understand the A dependence of heavy target data, a wealth of information on production cross sections will be essentially inaccessible. This problem and that of the poorly known charmed baryon branching fractions remain the most pressing today.

PT dependence

The one area in which there seems to be general agreement is that of the $p_{T}$ dependence of charm hadroproduction. The standard parameterization for this is,

$$
d \sigma / d p_{T}^{2} \alpha \exp \left(-b p_{T}^{2}\right)
$$


Table 2 shows the results from several representative experiments. The most striking feature is that the average $\mathrm{p}_{\mathrm{T}}$ and slope are independent of the incident beam, beam energy, target and produced species. From this we can gather that

$$
\left\langle p_{T}\right\rangle \simeq 800 \mathrm{MeV} \text { and }\langle b\rangle \simeq 1.1\left(\mathrm{GeV}^{2}\right)^{-1}
$$

The average $p_{T}$ is then about twice that seen for $\pi$ and $k$ production.

$x_{F}$ dependence

Although there is a general consensus on the transverse momentum dependence, none exists for the longitudinal momentum. Much of the interest in this subject revolves around the question of forward charm production in which one of the outgoing charm particles would be expected to carry a valence quark from the beam. The reader is directed to the review of Kernan and Van Dalen (18) and the references therein for a full description of the various theories of charm production.

The importance of the of the longitudinal momentum stems as well from its impact on total cross sections for those experiments which cannot cover charm momenta down to $x_{F}$ of zero. If we adopt the parameterization that

$$
d \sigma / d x_{F} \propto\left(1-x_{F}\right)^{n}
$$


then Table 3 contains measured values of $n$ for a variety of experiments. As usual the bulk of these are from heavy metal targets, and one is left to guess what may be the effect of a nuclear target on the momentum spectrum of the outgoing charm. (This may be counted as another outstanding reason for us to solve the problem of nuclear production.) One point is, though, apparent from Table 3. All those experiments whose sensitivity extends to $x_{F}=0$ measure a softer charm spectrum than those with a range of $x_{F}$ Iimited to larger values. Such differences can manifest themselves as whopping big changes in the inferred total cross sections since $\sigma \alpha 1 /(n+1)$. The NA11 collaboration for example measured $n=0.8 \pm 0.4$ when the apparatus acceptance was 1 imited to $x_{F}>0.2 .(19)$ Their improved spectrometer extended their acceptance down to zero, and the slope became $n=2.9$. The moral of this story seems to be that the reliable measurements can only be those which encompass central production.

A possible explanation is that one is viewing some sort of leading particle effects. These were first invoked to attempt to reconcile the differing values for total charm cross sections that came from the di-lepton ( $100 \mu \mathrm{b}$. versus discrete channel experiments at the ISR ( $\sim 1$ mb.). $(20,21,22)$ Evidence for such leading particles was seen by the CFRS collaboration who measured $\mu^{ \pm}$production off an iron dump with $\pi^{-}$and p beams. $(23,24)$ In the $\pi^{-}$ 
(quark content: $d \bar{u}$ ) part, they measured an axcess of $\mu^{-}$ events as compared to $\mu^{+}$which could be interpreted as a leading $D^{O}-D^{-}$component (quark content:cī and $\bar{c} d$ respectively)). No such effect was seen in the proton running. In that case $\Lambda_{c}$ would be the leading particle as it carries two of the valence quarks of the incident proton. Even more striking evidence for leading particles comes from NA27. (25) Their running was also broken into $\pi^{-}$and proton parts. Examination of the $\pi$ produced $D$ sample gave a bad fit to a single $\left(1-x_{F}\right)^{n}$ distribution. A much better fit comes from a two component model with $80 \%$ of the production being central with $n=7.5$ and $20 \%$ forward with a much harder spectrum characterized by $n=0.7$. These distributions and the associated fits are shown in Fig. 3. When the same sample was divided into "leading" $D^{\prime} s, i . e . D^{-}$and $D^{O}$, and central ones, $D^{+}$and $D^{\circ}$, then fits to the two samples yielded,

$$
\begin{aligned}
& D^{-}, D^{0} \quad n=2.0_{-0.5}^{+0.6} \text { and }\left\langle x_{F}\right\rangle=0.232 \\
& D^{+}, D^{0} \quad n=7.0_{-1.2}^{+1.4} \text { and }\left\langle x_{F}\right\rangle=0.107
\end{aligned}
$$

Again there was no evidence for leading particles in the proton sample. The case for leading charmed mesons seems well established while leading baryons have yet to be confirmed. 


\section{A fow other topics}

\section{D* production}

One of the most interesting recent results was that of the UA-1 collaboration's report of the obeservation of charmed mesons in jets at $\sqrt{s}=540 \mathrm{GeV}$. The signal was isolated by demanding that the mass of a $K \pi_{1} \pi_{2}$ combination minus the $K \pi_{1}$ be equal to a $\pi$ mass. This is the standard trick which uses the small $Q$ value of the $D^{*} \rightarrow D \pi$ decay. Their published result (26) found that the fragmentation of the charmed quark was softer than that seen in $e^{+} e^{-}$collisions and that $N\left(D^{* \pm}\right) /$ jet $=0.65 \pm 0.2$ (stat.) \pm 0.33 (sys.). This would have seemed to imply that there is on average one charm per jet at SPPS energies, a truly heroic charm production cross section. As has been remarked at this conference earlier, a reanalysis of the data has yielded $N\left(D^{* \pm}\right) /$ jet $=0.08 \pm 0.02$ (stat.) \pm 0.04 (sys.) which is more in keeping with conventional theoretical calculations. (27)

Both NA11 and NA27 ( $\pi^{-}$data) have published $D^{*}$ results as well. The two give compatible results. Both find $D^{*}$ production to be central with $n=4.3_{-1}^{+1.5}$ from the LEBC result (see Fig. 4) which goes down to $x_{F}=0$. (14) NA11 finds the smaller value of $n=3.2 \pm 1.5$, but they are limited to $x_{F}>0.2$ which may help explain the discrepancy. In any event, the two are equal within errors. The other question of interest 
is that of the $D^{*} / D$ ratio, the measure of indirect to direct D production. The findings are as follows,

$$
\begin{gathered}
D^{*} / D=0.9_{-0.6}^{+3.1} \\
D^{*} / D^{0}=1.00 \pm 0.25 \pm 0.12 \\
D^{*} / D^{ \pm}=0.48 \pm 0.22 \pm 0.15
\end{gathered}
$$

As noted in Ref. 14, because of the central nature of $D^{*}$ production, it cannot account for the forward component seen in the $\pi$ data.

\section{B production}

As alluded to earlier, only one experiment has presented evidence for hadronically produced B mesons, WA75. The apparatus used an emulsion for vertex detection coupled with a muon spectrometer. There was no other momentum analysis. The event in question is notable because the identification is done on topological grounds. Figure 5 shows the event in question. There are two muons in the event with one coming from a charged decay into a muon and a neutral 4 prong which is found downstream of this semi-leptonic decay vertex. This is interpreted as a $\mathrm{B}^{-}$. The other muon comes about from the cascade decay of $\bar{B}^{\circ} \rightarrow D^{-}+X$ with the second muon coming from the subsequent decay of the $D^{-}$. Identification 
of the $D^{-}$in this case is done on the basis of the $p_{T}$ of the muon which is too large to have come from a $K$ or $\pi$ decay. The cross section is $\sim 10 \mathrm{nb}$. The most interesting feature of the event is the short B lifetime that can be inferred. Whereas the world average (based solely on $e^{+} e^{-}$results) is $\tau_{B}=11.1 \pm 0.16 \times 10^{-13} \mathrm{~s} .(28)$, the average found from these two candidates is $\left\langle\tau_{B}\right\rangle=3_{-1}^{+2} \times 10^{-13} \mathrm{~s}$. This is a very intriguing result. It emphasizes the necessity for fixed target results on $B$ mesons. The only advantage that fixed target has over colliders is the ability to "sit on top" of the primary and secondary vertices, and it behooves us to make use of it.

\section{Conclusions}

After 10 years of hadroproduction experiments, we seem to know some things well. These include: the production parameters of $D^{\prime} s$ and $D^{*}$ 's; the $P_{T}$ dependence of charm production; and that there are leading particle effects in meson beams. What we do not know well are: $\sigma_{C}$ as a function of energy; the production ratio of $F^{ \pm}$to $D$; the charmed baryon sector; and finally how nuclear target data relate to production on protons.

What is needed for charm production is clean, reliable data at Tevatron energies with production on hydrogen and 
good vertex detection. To which goal, I submit, less than humbly, Fermilab experiment E743, an experiment which utilizes the Fermilab MPS for tracking and LEBC for vertex detection. The combination of chamber and spectrometer is virtually the same as the LEBC-EHS combination which has provided the most reliable production results to date.

\section{Acknow ledgements}

I would like to thank the organizing committee and the Aspen Center for Physics for their hospitality during my stay here. As always my E743 collaborators were invaluable in their efforts and assistance to me. In addition, I would like to thank the Aspen Valley Hospital for putting me back together in time to give this talk. Support for this work was provided by U.S. Dept. of Energy. 


\section{Reforences}

1. J.P. Albanese et al., Phys. Lett. 158B, 186 (1985).

2. D. Drijard et al., Phys. Lett. 81B, 250 (1979).

3. M. Aguilar-Benitez et. al., Phys. Lett. 122B, 312 (1983).

4. R. Bailey et al., Z. Phys. 28C, 357 (1985).

5. R. Bailey et al., Phys. Lett. 139B, 320 (1984).

6. K.L. Giboni et al., Phys. Lett. 85B, 437 (1979).

7. D. Drijard et al., Phys. Lett. 85B, 452 (1979).

8. S.F. Biagi et al., Phys. Lett. 150B, 230 (1985).

9. S.F. Biagi et al., Z. Phys. 28C, 175 (1985).

10. M.E. Duffy et al., Phys. Rev. Lett. 55, 1816 (1985).

11. M.C. Touboul, EHS Internal Note 85-14. The E613 results were communicated by R.C. Ball, Univ. of Michigan.

12. D.S. Barton et al., Phys. Rev. D27, 2580 (1983).

13. M.G. Abreu et al., CERN/EP 84-51.

14. C. Caso, CERN/EP 85-180.

15. C.H. Georgiopoulos et al., Phys. Lett.152B, 428 (1985).

16. M. Aguilar-Benitez et al., Phys. Lett. 156B, 444 (1985).

17. A.N. Aleev et al., Z. Phys. 23C, 333 (1984).

18. A. Kernan and G. VanDalen, Phys. Rep. 106, 297 (1984).

19. R. Bailey et al., Phys. Lett. 132B, 237 (1983).

20. A.G. Clark et al., Phys. Lett. 78B, 339 (1978).

21. L. Baum et. al., Phys. Lett. 68B, 279 (1977).

22. W. Lockman et al., Phys. Lett. 85B, 443 (1979).

23. A. Bodek et al., Phys. Lett. 113B, 77 (1982).

24. A. Bodek, Proc. XIV International Symposium on Multiparticle Dynamics Conf., Lake Tahoe. 
25. M. Aguilar-Benitez, submitted to Phys. Lett.

26. G. Arnison et al., Phys. Lett 147B, 222 (1984).

27. K. Ellis, Fermilab, private communication.

28. K. Berkelman, Cornell, these proceedings. 
Table 1: Measured DD cross sections for $x_{F}>0$

\begin{tabular}{|c|c|c|c|}
\hline Experiment & $\begin{array}{l}\text { Beam/ } \\
\text { Target }\end{array}$ & $\begin{array}{l}\sqrt{\mathrm{s}} \\
(\mathrm{GeV})\end{array}$ & $\begin{array}{l}\sigma \\
(\mu \mathrm{b} . / \text { hucleon })\end{array}$ \\
\hline CHARM $^{1}$ & $\mathrm{p} / \mathrm{Cu}$ & 27 & $9.4 \pm 3.1$ \\
\hline \multirow[t]{2}{*}{$\mathrm{E} 595^{1}$} & $\pi^{-} / \mathrm{Fe}$ & 24 & $17.5_{-3.9}^{+5.5}$ \\
\hline & $\mathrm{p} / \mathrm{Fe}$ & 27 & $10.7 \geq 1.1 \pm 1.8$ \\
\hline $\mathrm{E} 613^{1}$ & $p / W$ & 27 & $7.8 \pm 1.7$ \\
\hline \multirow[t]{2}{*}{ NA16 } & $\pi^{-} / p$ & 26 & $12.2_{-3.8}^{+7.6}$ \\
\hline & $p / p$ & 26 & $15.6_{-4.6}^{+8.2}$ \\
\hline NA18 ${ }^{1}$ & $p /$ Freon & 25 & $25 \pm 11$ \\
\hline NA27 & $\begin{array}{l}\pi^{-} / \mathrm{p} \\
\mathrm{p} / \mathrm{p}\end{array}$ & $\begin{array}{l}26 \\
27\end{array}$ & $\begin{array}{l}7.9 \pm 1.3 \\
11.2 \pm 2.3\end{array}$ \\
\hline $\begin{array}{r}\mathrm{R} 407 \\
408\end{array}$ & $p / p$ & 53 & $100-500 \pm 60 \%$ \\
\hline $\mathrm{R} 415^{2}$ & $p / p$ & 62 & $120-350 \pm 40 \%$ \\
\hline $\mathrm{R} 416^{2}$ & $p / p$ & 62 & $100-150 \pm 50 \%$ \\
\hline
\end{tabular}

2 Assuming a nuclear dependence of $A^{1}$.

Results are highly model dependent. 
17

Table 2: $P_{T}$ dependence of charm production. (see Eq. 2)

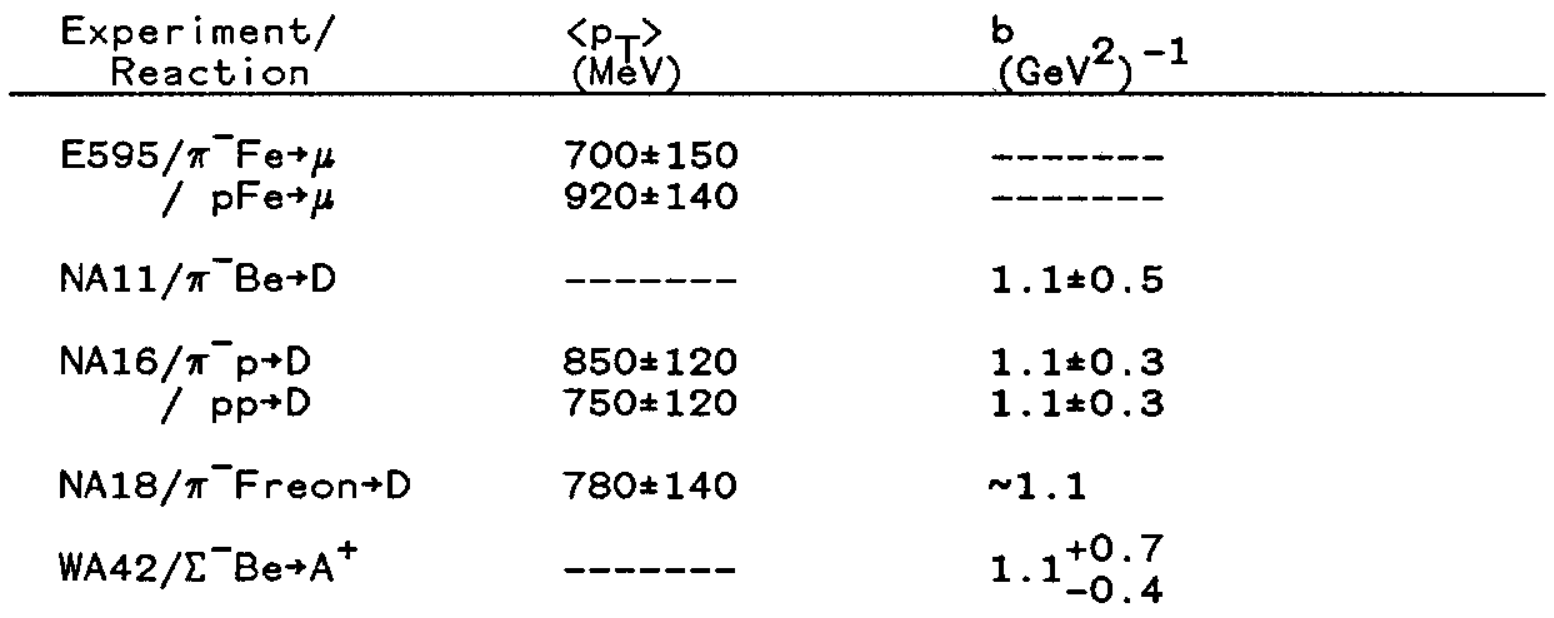


Table 3: Feynman $x$ dependence of charm production parameterized by Eq. 3 .

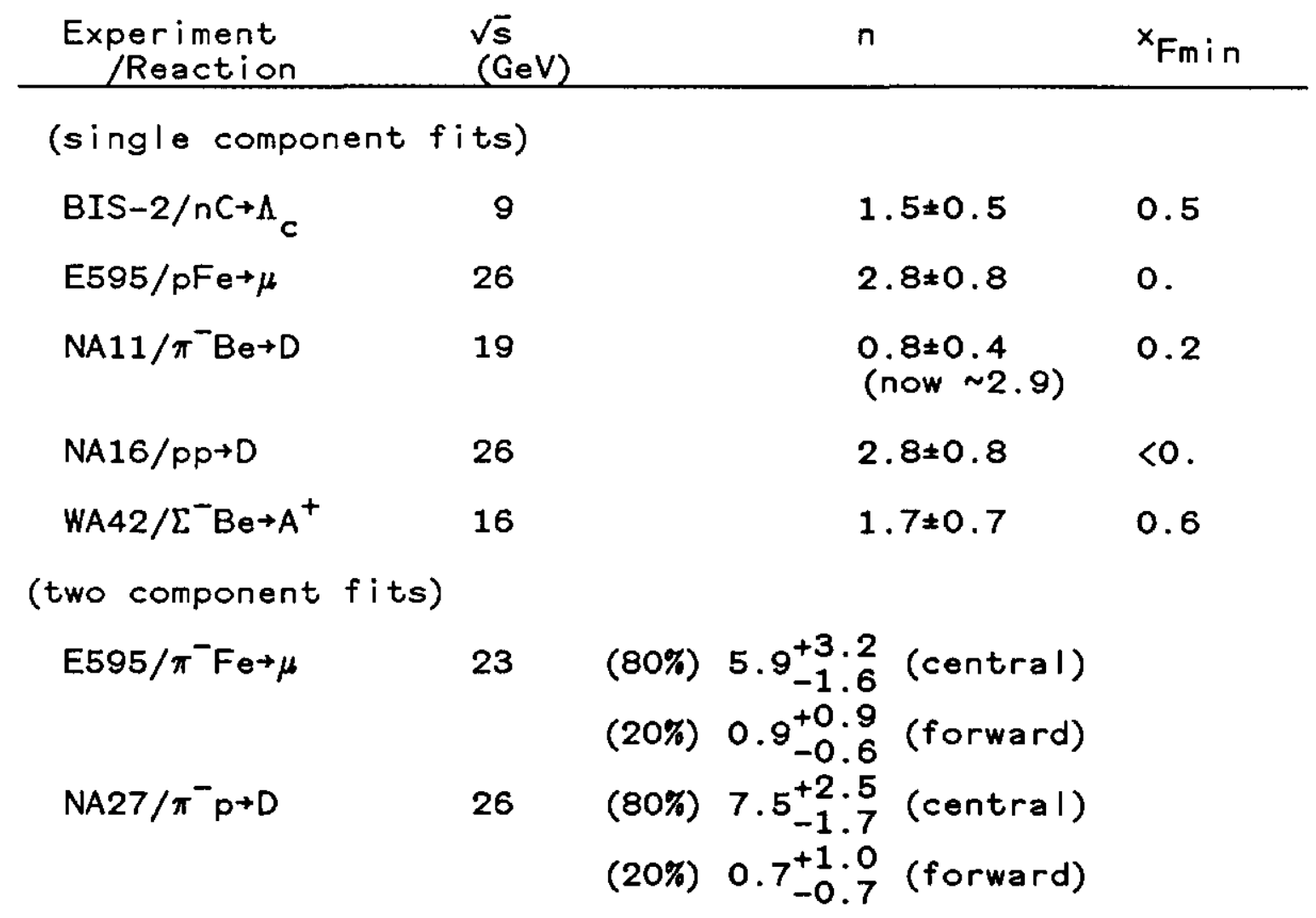




\section{Figure Captions}

Fig. 1: The nuclear dependence parameter, $\alpha$, as a function of $x_{F}$ as given by Barton et al. in Ref. 12 .

Fig. 2: The observation of the decay $D^{ \pm} \rightarrow \phi \pi^{ \pm}$as reported by the E623 collaboration (Ref. 15). No evidence is seen for the decay of the $F^{ \pm}$into this same final state.

Fig. 3: $x_{F}$ distributions for D mesons from NA27 (Ref 14). (a) All D's. The continuous $l i n e$ is a two component fit, whereas the the dotted and dashed lines are single component fits to two different production models. See the text for details.

(b) Leading $D^{\prime} s\left(i . e . D^{-}, D^{0}, D^{*-}\right.$ ) only. The solid line is from the two component fit of (a).

(c) Non-leading $D^{\prime} s\left(D^{+}, D^{0}, D^{*+}\right)$. The solid line comes from the two component fit of (a).

Fig. 4: Fit to the $x_{F}$ distributions of $D^{*}$ mesons from NA27 (Ref. 14). The production is seen to be central. The fit could only be done for charged $D^{*}$ 's as seen in (a), but the value found for $n$ is seen to be compatible with the neutral $D^{*}$ 's as seen in (b) 
Fig. 5: The first observation of $B$ meson production in a hadron induced reaction. The identification depends on the large transverse momenta of the two muons in the event and the topology of the decay of the daughter D at vertex 2 . Further details can be found in the text and Ref. 1 . 


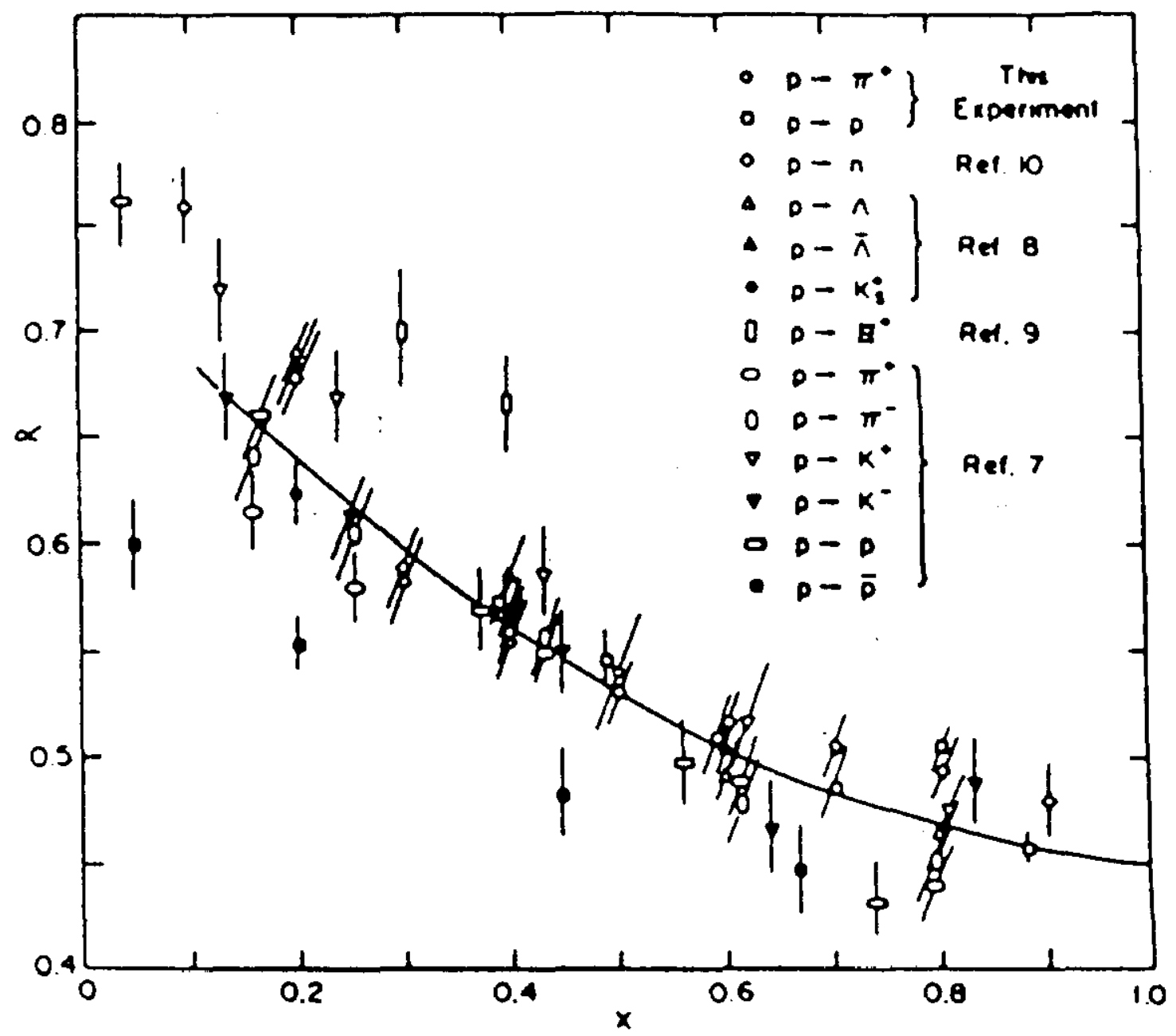

Fig. I 


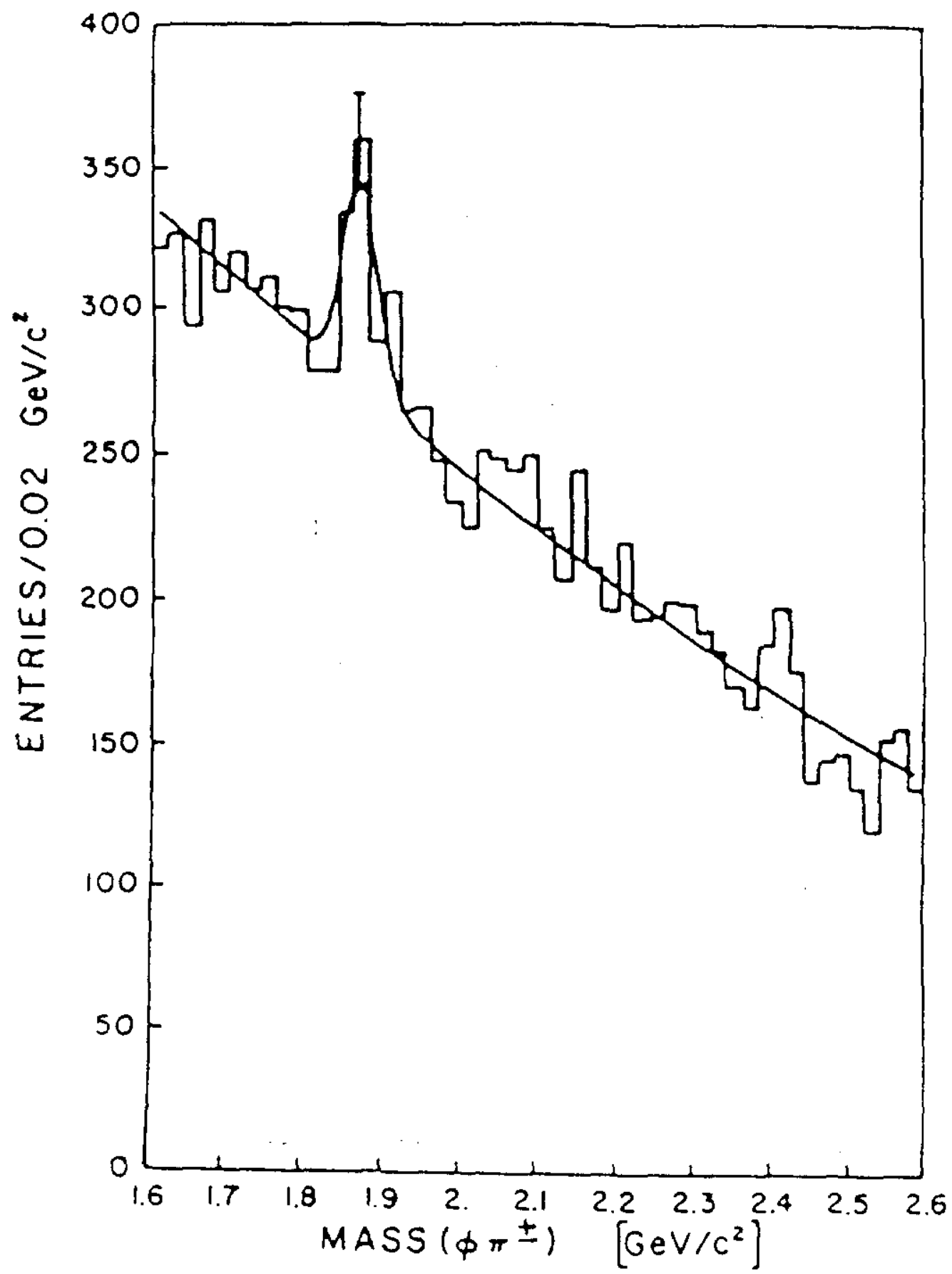

Fig. 2 


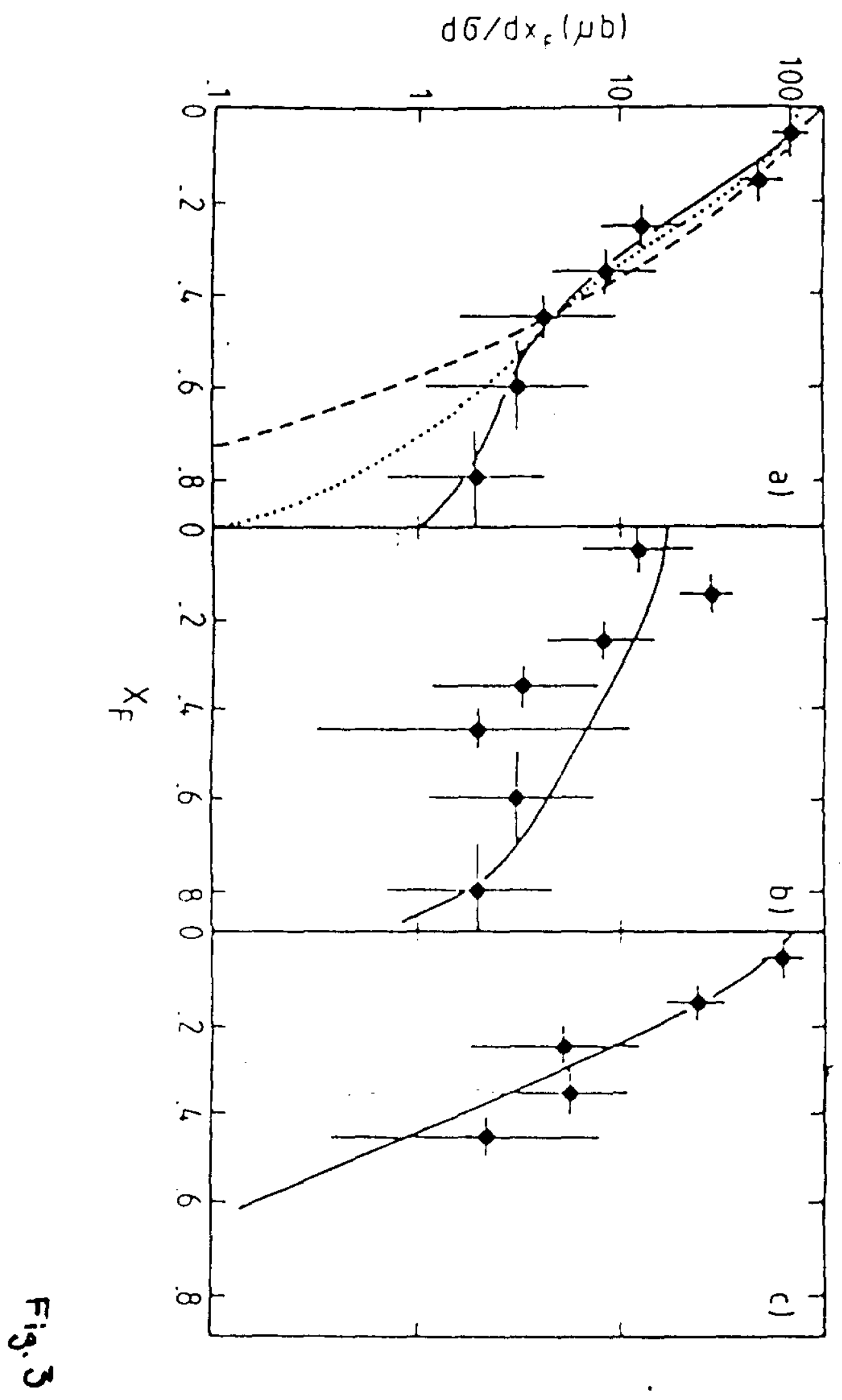




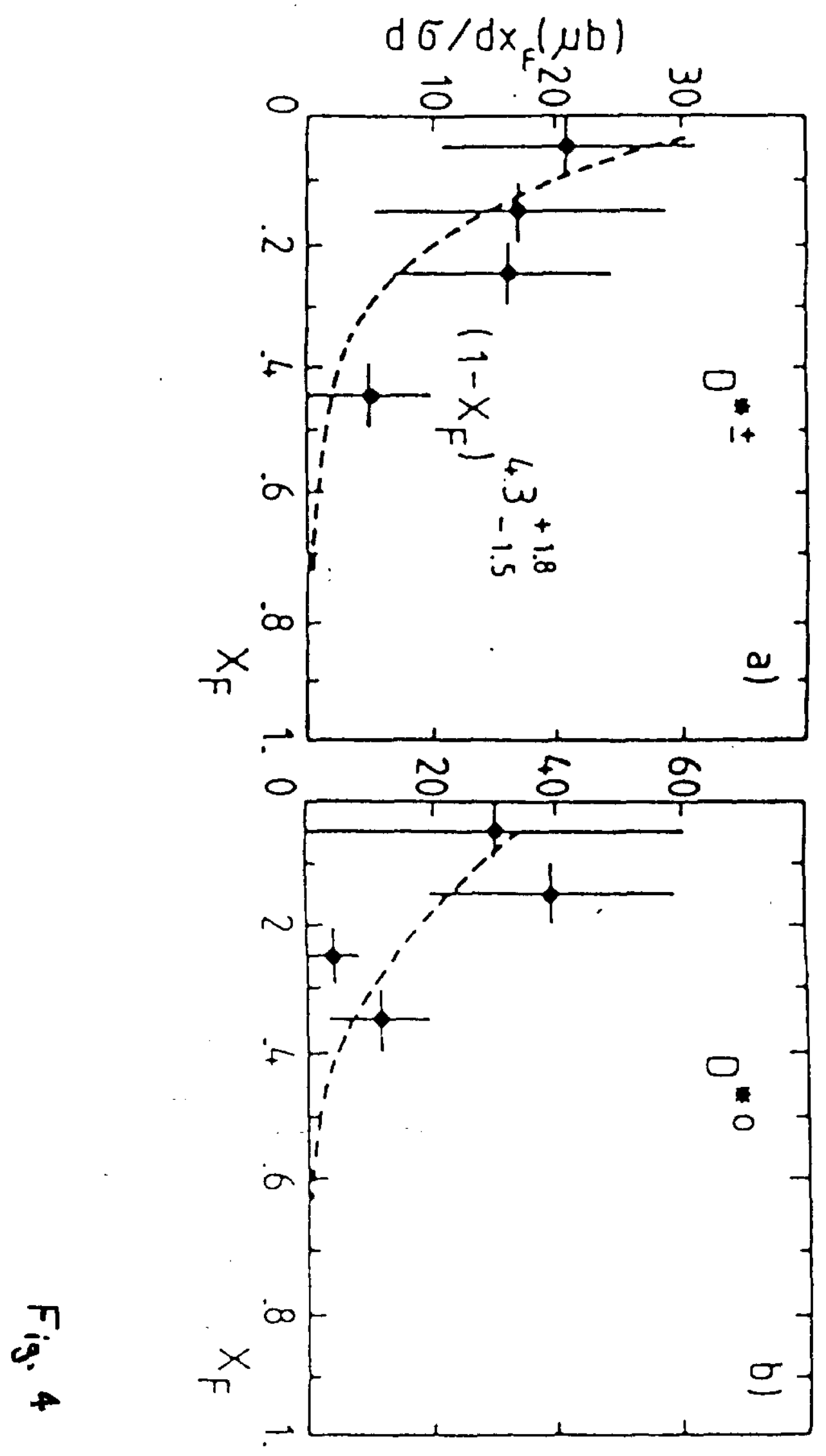




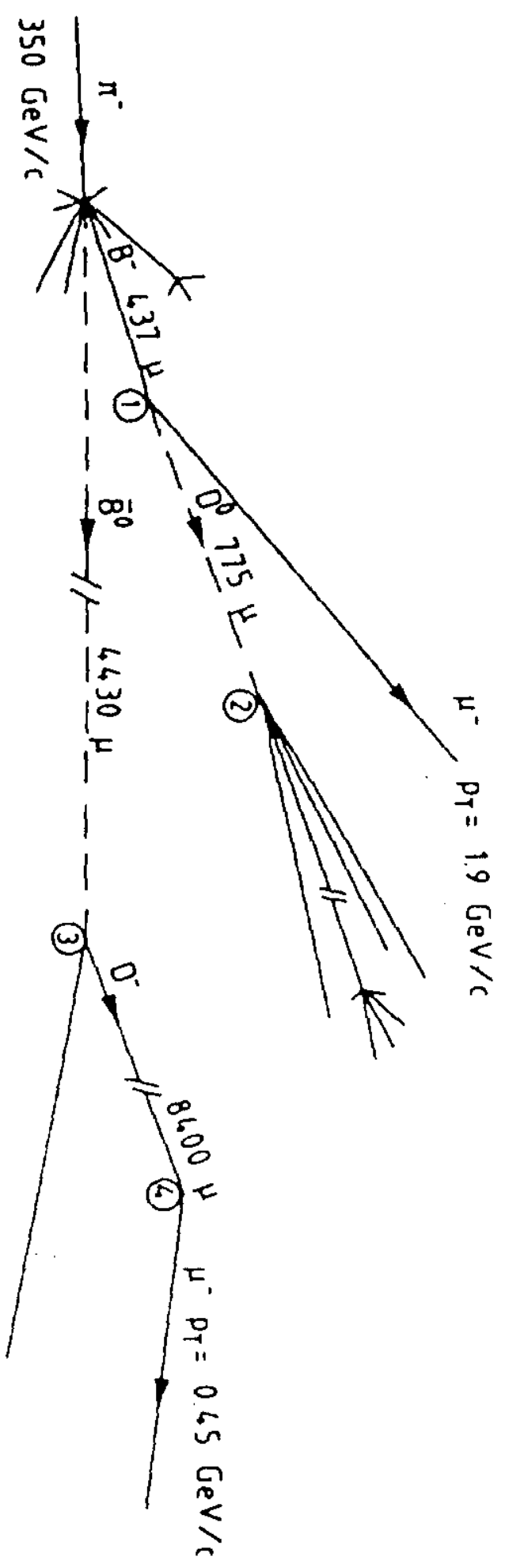

